\title{
SOME REMARKS ON NILPOTENT GROUPS WITH ROOTS
}

\author{
GILBERT BAUMSLAG ${ }^{1}$
}

1. Let $\boldsymbol{\omega}$ be a nonempty set of primes. Then a group $G$ is called an $E_{\varpi}$-group if the equation

$$
x^{p}=g
$$

is soluble for every $p \in \varpi$ and every $g \in G$. Similarly we call a group $G$ a $D \varpi$-group if the equation (1) (above) is not only soluble, but uniquely soluble. The class of $E_{\varpi}$-groups and the class of $D \varpi$-groups will be denoted respectively by $E_{\boldsymbol{\sigma}}$ and $D_{\boldsymbol{\sigma}}$. Clearly $E_{\boldsymbol{\sigma}} \supset D \varpi$. There is a less trivial relationship between these two classes of groups. If we denote by $\Theta(C)$ the class of groups which occur as homomorphic images of the members of a given class of groups $C$, then $\Theta(D \varpi)=E \varpi$ (see G. Baumslag [1]).

Now suppose that $N$ denotes the class of nilpotent groups. One of the consequences of our results in this note is the fact that $\Theta(D \varpi \cap N)=E_{\varpi} \cap N$. However we shall show that if $L$ is the class of locally nilpotent groups then $\Theta(D \varpi \cap L) \neq E_{\varpi} \cap L$. This leads us to ask whether, perhaps, $\Theta(D \varpi \cap S) \supseteq E_{\varpi} \cap L$, where $S$ here is the class of locally soluble groups.

2. Let $G$ be a nilpotent group of class $c$. The subset $X$ of $G$ is said to freely generate $G$ if

(i) $X$ generates $G$, and

(ii) for every group $H$ which is nilpotent of class at most $c$ and every mapping $\theta$ of $X$ into $H$ there exists a homomorphism $\phi$ of $G$ into $H$ which coincides with $\theta$ on $X$. A nilpotent group of class $c$ which is freely generated by some set is called a free nilpotent group. These groups are simply isomorphic copies of $F / F_{c+1}$, where $F$ is some free group and $F_{c+1}$ is the $(c+1)$ st term of the lower central series of $F$.

We shall embed a free nilpotent group $G$ in a nilpotent $D \varpi$-group $G^{*}$. This embedding has already been carried out in a rather more general context by A. I. Mal'cev [5]; however he makes use of Lie ring methods and so we prefer to carry out here a different direct embedding procedure which lends itself more easily to the application we have in mind.

Suppose then that $G$ is a free nilpotent group of class $c$ freely generated by the set $X$. Put $G=G_{1}$ and $X=X_{1}$. Suppose now that for each

Received by the editors March 30, 1960.

1 This work was supported in part by National Science Foundation Grant G-9665. 
positive integer $n G_{n}$ is a free nilpotent group freely generated by $X_{n}$, with

$$
\left|X_{n}\right|=\left|X_{n-1}\right| \quad(n=2,3, \cdots),
$$

where $|S|$ denotes the cardinality of the set $S$. Let

$$
p_{1}, p_{2}, \cdots, p_{n}, \cdots
$$

be an infinite sequence of primes in $\boldsymbol{w}$, chosen so that for any $p \in \varpi$ and any positive integer $N$ there exists an integer $M \geqq N$ such that $p_{M}=p$. Now define

$$
H_{n}=g p\left(x^{p_{n-1}} ; x \in X_{n}\right) \quad(n \geqq 2) .
$$

By a theorem of G. Baumslag [2], $H_{n}$ is nilpotent of class $c$ and is freely generated by

$$
X_{n}^{p_{n-1}}=\left\{x^{p_{n-1}} \mid x \in X_{n}\right\} .
$$

Clearly $\left|X_{n}^{p_{n-1}}\right|=\left|X_{n}\right|=\left|X_{n-1}\right|$. Hence we may identify $G_{n-1}$ with $H_{n}$ for $n=2,3, \cdots$. Finally we put

$$
G^{*}=\bigcup_{n=1}^{\infty} G_{n}
$$

Clearly $G^{*}$ is nilpotent of class $c$. By the choice of the sequence (3) every element of $G^{*}$ can be written as a product of $p$ th powers for any $p \in \boldsymbol{~}$. Consequently, by a theorem of S. N. Cernikov (see Kuroš $\left[4\right.$, vol. 2, p. 238]), every element of $G^{*}$ has a $p$ th root for each $p \in w$. Moreover $G^{*}$ is clearly torsion-free, since each $G_{n}$ has this property. Therefore as extraction of $p$ th roots in a torsion-free nilpotent group is unique whenever it is possible (A. I. Mal'cev, see e.g. Kuroš [4, vol. 2 , p. 247]), $G^{*}$ is a $D \varpi$-group. In the following theorem we adopt the notation of this paragraph.

THEOREM 1. Suppose $\sigma$ is a set of primes such that $\sigma \geqq \omega$. Then for every $E_{\sigma}$-group $H$ which is nilpotent of class at most $c$ and every mapping $\theta$ of $X$ into $H$ there exists a homomorphism $\phi$ of $G^{*}$ into $H$ which coincides with $\theta$ on $X$.

Proof. Since $G$ is freely generated by $X$ we can extend $\theta$ to a homomorphism $\theta_{1}$ of $G$ into $H$. Suppose, inductively, that $\theta_{i}$ is a homomorphism of $G_{i}$ into $H$. Now $\theta_{i}$ is determined by its effect on $X_{i}$, the free set of generators of $G_{i}$. Suppose $y \in X_{i}$. Then, by our construction of $G^{*}$, 
We put

$$
x \theta_{i+1}=z,
$$

where $z$ is chosen to be any $p_{i}$ th root of $y \theta_{i}$. Thus

$$
\left(x \theta_{i+1}\right)^{p_{i}}=z^{p_{i}}=y \theta_{i} \text {. }
$$

In this way we define the effect of a mapping $\theta_{i+1}$ on the free generators $X_{i+1}$ of $G_{i+1}$ and, since $X_{i+1}$ is a free set of generators, $\theta_{i+1}$ can be extended to a homomorphism of $G_{i+1}$ into $H$, which we again denote by $\theta_{i+1}$. It follows from (4) that $\theta_{i+1}$ extends $\theta_{i}$. Finally we define

$$
\phi=\bigcup_{i=1}^{\infty} \theta_{i} ;
$$

i.e., if $g \in G^{*}$, then we define $g \phi=g \theta_{n}$ if $g \in G_{n}$. Clearly $\phi$ is a homomorphism of $G^{*}$ into $A$. Furthermore, by its very definition $\phi$ coincides with $\theta$ on $X$. This completes the proof.

Corollary. $\Theta(D \varpi \cap N)=E_{\varpi} \cap N$.

Proof. Let $H \in E_{\varpi} \cap N$. Then $H$ is nilpotent of, say, class $c$. Choose $X$ so that

$$
|X|=|B|
$$

and let $G^{*}$ be as above. Define $\theta$ to be any mapping from $X$ onto $H$, whose existence is assured by (5). By Theorem 1 , this mapping can be extended to a homomorphism of $G^{*}$ onto $H$. The corollary therefore follows.

It is worth pointing out that the two classes of groups $D \varpi \cap N_{c}$, $E_{\varpi} \cap N_{c}$, where $N_{c}$ is the class of groups which are nilpotent of class at most $c$, both form varieties of algebras in the sense of $\mathrm{P}$. Hall; in other words they are equationally definable. The group $G^{*}$ is simply one of the free algebras of the variety $D \varpi \cap N_{c}$. Thus, on completely general grounds, any mapping of $X$ into a group $H$ which belongs to $D_{\varpi} \cap N_{c}$, can be extended to a homomorphism of $G^{*}$ into $H$. This statement, however, does not immediately imply the corresponding one if $H \in E \varpi \cap N_{c}$.

Let us call an integer $m>0$ an $\varpi$-number if the prime divisors of $m$ belong to $\approx$. The following theorem then holds.

Theorem 2. Let $G \in D \varpi \cap L$, i.e., suppose $G$ is a locally nilpotent 
group in $D \varpi$, and let $N$ be a normal subgroup of $G$. Then the elements in $G / N$ whose orders are $\varpi$-numbers form a subgroup contained in the center of $G / N$.

Proof. It is well known that in a locally nilpotent group the elements whose orders are $\varpi$-numbers form a subgroup (cf., e.g., Kuroš $\left[4\right.$, vol. 2, p. 216]). It remains to prove, therefore, that if $g^{m} \in N$, where $g \in G$ and $m$ is an $\varpi$-number, then $[g, h] \in N$ for every $h \in G$.

Since $G$ is locally nilpotent, $g$ and $h$ generate a nilpotent subgroup of $G$. Let $H$ be a subgroup of $G$ containing $g$ and $h$ which is nilpotent and also belongs to $D_{\boldsymbol{w}}$ - the existence of such a subgroup $H$ follows e.g. by a theorem of G. Baumslag [1]. Since

$$
H N / N \cong H / H \cap N,
$$

$H N / N$ is nilpotent. Clearly $H N / N$ then belongs to $E_{\varpi}$, since $H \in E_{\varpi}$. Now in a nilpotent group in $E_{\varpi}$ the elements whose orders are $\varpi$ numbers belong to the centre (see [1]). In particular $g N$ belongs to the centre of $H N / N$, i.e., $[h, g] \in N$. Since $h$ was arbitrary, the theorem follows.

Now every finite $p$-group can be embedded in a locally nilpotent $E_{\boldsymbol{\sigma}}$-group (see G. Baumslag [3]). Consequently there are nonabelian locally nilpotent $E_{\varpi}$-groups which are periodic. This would, however, not be the case if $\Theta(D \varpi \cap L)=E_{\varpi} \cap L$, by Theorem 2 . Thus we have proved

Theorem 3. $\Theta\left(D_{\varpi} \cap L\right) \neq E_{\varpi} \cap L$.

One is tempted to enquire, at this point, whether there is a class $C$ of groups with $C$ properly contained in $D_{\varpi}$, such that

$$
\Theta(C) \supseteq E_{\varpi} \cap L .
$$

3. It is perhaps of interest to make some further remarks concerning the group $G^{*}$ of $\S 2$. Let us call a subgroup of $G^{*}$ which is at the same time a $D \varpi$-group, an $\varpi$-subgroup. We say that a subset $Y$ of $G^{*}$ is a free $\varpi$-generating set of $G^{*}$ if

(i) $Y \varpi$-generates $G^{*}$, i.e., the smallest $\varpi$-subgroup of $G^{*}$ containing $Y$ is $G^{*}$ itself, and

(ii) for every $D_{\varpi}$-group $H$ which is nilpotent of class at most $c$, and every mapping $\theta$ of $Y$ into $H$, there exists a mapping $\phi$ of $G^{*}$ into $H$ which coincides with $\theta$ on $Y$.

The following theorem then holds (again we assume the notation of §). 
Theorem 4. Let $Y$ be a subset of $G^{*}$. Suppose $|X|=|Y|$ and that $|X|$ is finite. If $Y$ w-generates $G^{*}$, then $Y$ is a free $\varpi$-generating set of $G^{*}$. Further, if $F=g p(Y)$, then $F$ is a free nilpotent group of class $c$ which is freely generated by $Y$.

We need the following lemma for the proof of Theorem 4 .

Lemma. Let $A$ be a nilpotent group of class $c$ which can be generated by $s(<\infty)$ elements. Suppose that $B$ is a subgroup of $A$ which is nilpotent of class $c$ and is freely generated by $\left\{b_{1}, b_{2}, \cdots, b_{s}\right\}$. Then $A$ is a free nilpotent group.

Proof. Suppose $A=g p\left(a_{1}, a_{2}, \cdots, a_{s}\right)$. Let $\theta_{1}$ be the homomorphism of $B$ onto $A$ defined by

$$
b_{1} \theta_{1}=a_{1}, b_{2} \theta_{1}=a_{2}, \cdots, b_{s} \theta_{1}=a_{s} .
$$

Let $N_{1}$ be the kernel of $\theta_{1}$ :

$$
B / N_{1} \cong A \text {. }
$$

If $N_{1}=1$, then $A \cong B$ as required. If $N_{1} \neq 1$, then by first carrying out the same procedure with $B / N$, which contains a free nilpotent subgroup isomorphic to $A$, and then iterating this process, we obtain a series

$$
1<N_{1}<N_{2}<\cdots
$$

which is defined in the obvious way; thus we have a properly ascending series of subgroups of the finitely generated nilpotent group $A$. This is impossible by a theorem of K. A. Hirsch (see e.g., Kuroš [4, vol. 2, p. 193]) and so the proof of the lemma is complete.

To prove Theorem 4, we begin by noting (see Kuroš $[4$, vol. 2, p. 249]) that as $Y \varpi$-generates $G^{*}$, there exists an $\varpi$-number $m$ such that $x^{m} \in F$ for each $x \in X$. Thus, by the lemma, $F$ is a free nilpotent group of class $c$ freely generated by $Y$. We put $Y_{1}=Y, F_{1}=F$ and having defined $Y_{n}$ so that $Y_{n}$ freely generates $F_{n}=g p\left(Y_{n}\right)$, we define (see (3)):

$$
Y_{n+1}=\left\{x \mid x^{p_{n}}=y \text { for some } y \in Y_{n}\right\} .
$$

Then $\left|Y_{n+1}\right|=|Y|$. Furthermore, if we put $F_{n+1}=g p\left(Y_{n+1}\right)$, by the lemma, it follows that $F_{n+1}$ is free nilpotent of class $c$ freely generated by $Y_{n+1}$. Finally we put $F^{*}=\bigcup_{i=1}^{\infty} F_{i}$; then by the choice of the sequence (3), every element of $F^{*}$ is a product of $p$ th powers for every $p \in \varpi$. So, invoking Cernikov's theorem again, $F^{*}$ is a $D \varpi$-group. So $F^{*}=G^{*}$. It follows now by its very construction that $F^{*}\left(=G^{*}\right)$ is 
freely $\varpi$-generated by $Y$ (cf. the proof of Theorem 1 ). So the proof of Theorem 4 is complete.

We remark, in conclusion, that the lower central series of the group $G^{*}$ has a quite analogous structure to that of the lower central series of a free nilpotent group of rank $|X|$. The details involve the use of basic commutators and so, in order to keep this note self-contained, we prefer to omit the proof of this statement until a later date.

\section{REFERENCES}

1. Gilbert Baumslag, Groups with unique roots, to appear in Acta Math.

2. - On free polynilpotent subgroups of free polynilpotent groups. Unpublished.

3. - Wreath products and p-groups, Proc. Cambridge Philos. Soc. vol. 55 (1959) pp. 224-231.

4. A. G. Kuros, Theory of groups, vols. I and II, New York, Chelsea Publishing Co., 1956.

5. A. I. Mal'cev, Nilpotent torsion-free groups, Izv. Akad. Nauk SSSR. Ser. Mat. vol. 13 (1949) pp. 201-212.

Princeton University 